\title{
A low cost auto-filling and refrigeration rate regulated liquid nitrogen controller for near infrared instruments
}

\author{
Narsireddy Anugu ${ }^{1}$, Antonio Amorim², Paulo Garcia ${ }^{1}$, \\ Paulo Gordo ${ }^{2}$, Tiago Frederico², Jorge Abreu² \\ ${ }^{1}$ SIM-Faculdade de Engenharia da Universidade do Porto, 4200-465 Portugal; ${ }^{2} \mathrm{SIM}$ - \\ Faculdade de Ciências da Universidade de Lisboa, 1749-016, Portugal; \\ (narsireddy.anugu@fe.up.pt)
}

\begin{abstract}
Liquid Nitrogen is one of the key refrigerating elements in cooling near infrared science instruments to reduce the dark, readout noises and thermal emissions in the near infrared originated from the instrument structure. Usually, a small liquid nitrogen tank connected to the near infrared instrument is auto filled from a large Dewar in order to maintain required low temperatures during the experiment for several hours. The detectors used in these instruments are quite expensive and they need to be cooled down steadily $(<2 \mathrm{~K} / \mathrm{min})$ to avoid mechanical damage. The steady state cooling of the detector is the key requirement to be considered while cooling down the detector. In this paper, a controller is developed to auto-fill the liquid nitrogen tank and also to keep the refrigeration rate of the detector below $2 \mathrm{~K} / \mathrm{min}$. A systematic survey of auto-filling controllers is studied. The auto-filling of liquid nitrogen from Dewar to tank is implemented with a standard on-off controller. To address the critical refrigeration rate of the detector, two approaches are studied: a) by fixed time pumping; b) by feedback the detector cooling rate. In this work we have used inexpensive equipment to develop this controller. It is very successfully used for GRAVITY acquisition camera, a near infrared instrument for European Southern Observatory. This controller has been stable and efficient for our experiment. This low cost controller can be used for any student laboratory and research.
\end{abstract}

Subject Headings. Thermodynamics, Astronomy, Optics, Electronics.

Author Keywords. Liquid Nitrogen, Liquid Level Controllers, Refrigeration Rate, Beam Analyzing, Near Infrared Astronomy.

\section{Introduction}

Liquid Nitrogen $\left(\mathrm{LN}_{2}\right)$ is one of the most common cooling agents in near infrared astronomical instruments. For example, acquisition camera (Gordo et al. 2014) is a multiple telescope's near infrared beams analyzing instrument which is refrigerated with $\mathrm{LN}_{2}$. The advantages of keeping near infrared instruments at low temperatures are to minimize detector's readout and dark noises and it also reduces thermal emissions in the near infrared originated from the instrument structure. The acquisition camera, a sub instrument of GRAVITY(Eisenhauer et al. 2011), is designed to operate at $L_{2}$ temperatures. GRAVITY is a beam combiner instrument, which combines four very large telescopes $(8 \mathrm{~m})$ beams of European Southern Observatory (ESO) and delivers very precise astrometric measurements of stars positions in the vicinity of Milky Way Galactic Center supermassive Black Hole (Gordo et al. 2014). The acquisition camera instrument has been built (optical alignment and assembly) at room temperature. However, before it integrating within GRAVITY instrument, it requires a refrigeration system to validate its optical performance at $\mathrm{LN}_{2}$ temperature. 
The acquisition camera optical performance evaluating experiments at $\mathrm{LN}_{2}$ temperatures are usually carried out for a few days (Gordo et al. 2014). The optical experiments consist of mainly measuring how well the optics is focused and characterization of the system performance. During the course of the experiment, the instrument must be maintained at $\mathrm{LN}_{2}$ temperatures. It can be achieved by having a thermal connection between the acquisition camera to a cold finger of a $\mathrm{LN}_{2}$ tank within a cryostat (Carvas 2012). In order to reduce time and effort of filling the $\mathrm{LN}_{2}$ tank manually, it is automatically filled from large a $\mathrm{LN}_{2}$ Dewar. Detectors (ex: Hawaii 2RG) used for the infrared instruments are usually expensive and those need to be refrigerated steadily $(<2 \mathrm{~K} / \mathrm{min}$ rate) in order to ensure their safety. It is the critical requirement that needs to be taken care of while filling automatically the $\mathrm{LN}_{2}$ tank.

A lot of research has been reported on reliable and efficient auto-filling (Landis, Madden, and Goulding 1986; Gamblin, Goldberg, and Scag 1959; Murray and Atkinson 2004; Shuhua Ma et al. 2010; Morrell and Manning 1996; Zhao Yiding and Song Zengfeng 2010). Landis et al., Gamblin et al., Murray et al. and Morrel et al. describes an on-off control method, and Shuhua et al. \& Zhao et al. reports a proportional-integral-derivative (PID) based control method for auto-filling. The standard auto-filling controllers do not address the detector refrigeration rate $(R, R<2 K / \mathrm{min})$ during the cooling down process. However, commercially available Lakeshore cryogenic temperature controller (model 350) offers solution to the steady state refrigeration of the detector. This controller is equipped with heaters to warm the detector in order to keep the detector refrigeration rate below its critical limit. But these controllers are relatively expensive.

In this context, it was motivated to develop a low cost controller which can automatically fill $\mathrm{LN}_{2}$ tank efficiently by taking care of the detector refrigeration rate under control during cooling down process. As far as we know, a low cost detector refrigeration rate regulated auto filling system has not yet reported elsewhere, hence reported here.

The content of this paper is organized in three main sections. The Sec. 2 briefly summarizes the controller's concept and design. The designed controller hardware and software implementations are presented in Sec. 3 and 4.

\section{Control operation and design (auto-filled + detector refrigeration rate regulated)}

To auto-fill the $\mathrm{LN}_{2}$ tank, a standard on-off controller (Morrell and Manning 1996) is chosen for its low cost and easy implementation. In the on-off control method, $\mathrm{LN}_{2}$ is pumped for a time and stopped abruptly for another time. When pressure is applied to the Dewar, the liquid stored in it is transferred into the $\mathrm{LN}_{2}$ tank which is placed inside the cryostat via a $\mathrm{LN}_{2}$ delivery pipe. The flow is usually controlled by a solenoid valve (S). The on-off controller time periods are coded in a processor and the valve is opened and closed based on that processor signal. The operation of the on-off controller system is shown in Fig. 1 left panel.

The liquid level in the tank can be detected by mounting two temperature sensors within it. One is at the bottom $\left(A_{0}\right)$ of the tank and second one is at its inlet $\left(A_{1}\right)$ as shown in Fig. 1 . The temperature controller issues "ON" signal when a temperature sensor detects the temperature of $\mathrm{LN}_{2}$ (set point 77K). That is when it is fully submerged within $\mathrm{LN}_{2}$, otherwise it gives a zero voltage "OFF" signal. Utilizing these two sensor signals (ON/OFF states), liquid level in the tank can be estimated. Based on that, instructions are sent to the solenoid valve to transfer $L_{2}$ from Dewar to the tank. $L N_{2}$ transfer flow chart is presented in Table. 1. In the filling process, the liquid is allowed to flow until the inlet $\left(A_{1}\right)$ sensor is submerged in $L N_{2}$. 
In other words, when the valve is open and if the tank is fully filled, the control valve is automatically shut.

In order to keep the critical detector refrigeration rate under control $(<2 \mathrm{~K} / \mathrm{min})$ during the cooling down process, it is studied in two ways. Firstly, the detector refrigeration rate is calculated for every $1 \mathrm{~s}$ and feedback to the solenoid valve control. Secondly, the control valve is opened and closed for a fixed interval of time to fill the tank, in cycles, as a step function. The time period is chosen such that the detector refrigeration rate is always below $2 \mathrm{~K} / \mathrm{min}$ during the cooling down process.

\begin{tabular}{lccl}
\hline Sensor $A_{0}$ & Sensor $A_{1}$ & $\mathbf{L N}_{2}$ level & Filling state \\
\hline OFF & OFF & empty & fill \\
ON & OFF & partially filled & fill \\
ON & ON & full & don't fill \\
\hline
\end{tabular}

Table 1: The on-off control opens the solenoid valve to fill when the liquid level falls below the inlet sensor $\left(A_{1}\right)$

The time period is mainly dependent on pressure applied to Dewar in order to flow $\mathrm{LN}_{2}$, amount of liquid in the tank, diameter of the $\mathrm{LN}_{2}$ delivery pipe and the cold finger. The fixed time value can be measured in two ways. Firstly, it can be estimated using empirical calculation. The empirical time period calculation involves considering the specific heats of the material used in the system and liquid pumping flow rate (lit/s). Secondly, the fixed time can be calibrated from a cool down experiment. The calibration involves experimenting different time periods to obtain an optimized value. Once the time period is chosen, the hardware cannot be altered.

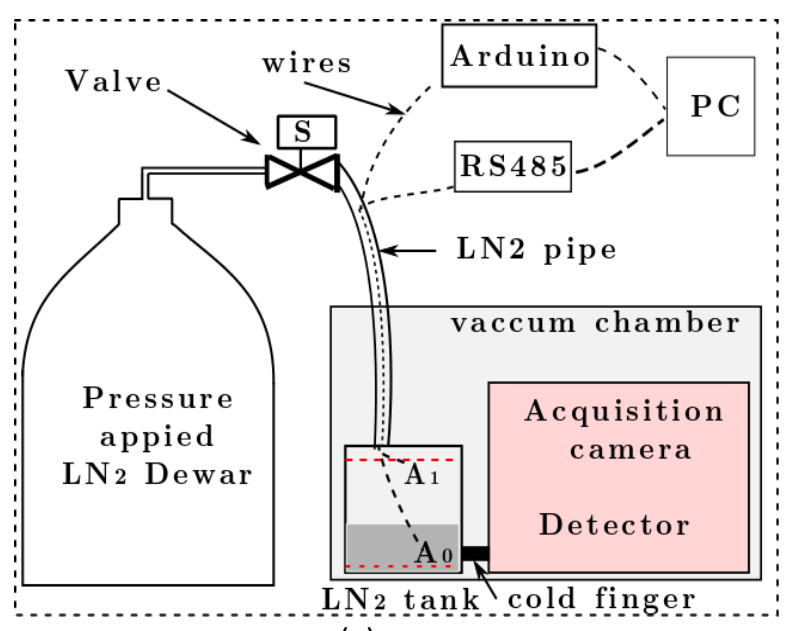

(a)

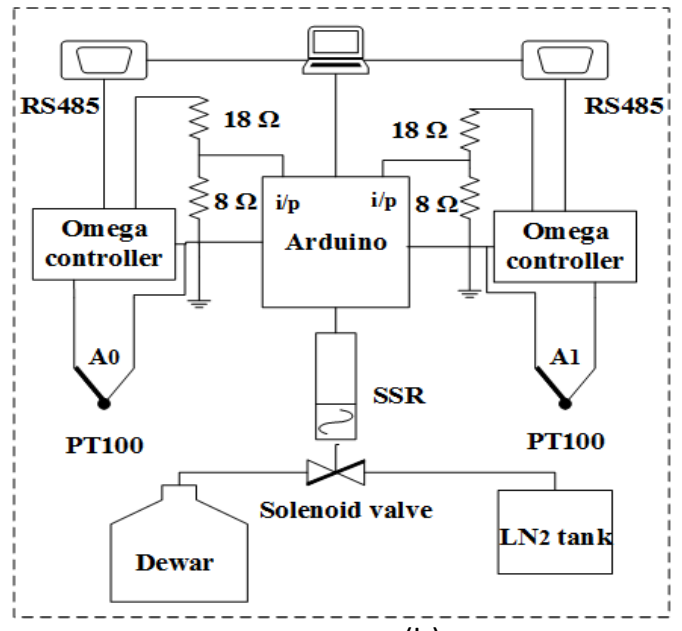

(b)

Figure 1: (a) LN2 auto filling from Dewar to tank is transferred via solenoid valve. (b) The circuit diagram of the controller. The valve is controlled by an Arduino by utilizing level detection sensors signals

The detector refrigeration rate controller performance is dependent on choosing appropriate fixed time period $(\mathrm{T})$ or refrigeration rate limit (R) properly, in the above mentioned algorithms. The refrigeration rate does not go to zero immediately when the valve is closed but it takes time to reach it. The reason is cooling inertia. Taking this effect into count, a fixed time period and a refrigeration rate limit parameters are essential to be appropriately employed in the control process. In this work, the fixed time period is evaluated from the calibration experiment as shown in Figure 2. The detector refrigeration rate limit is taken as $1.5 \mathrm{~K} / \mathrm{min}$. 


\section{Control Hardware}

The on-off controller hardware consists of a Dewar, a valve, a liquid carrying pipe, $\mathrm{LN}_{2}$ level detecting sensors in the tank, a solid state relay and a processor as indicated in Morrell and Manning 1996 and shown in Fig. 1 right panel. While choosing the hardware, preference is given for a simple circuit and for a low cost solution.

The acquisition camera vacuum cryostat is built in-house (Carvas 2012). A Dewar of 100 liters of capacity is already available was used. Two $\mathrm{LN}_{2}$ level detecting temperature sensors (PT100) are mounted in the $\mathrm{LN}_{2}$ tank. These sensors are connected to Omega controllers (CN7800) using $\mathrm{LN}_{2}$ insulated wires. The sensor temperatures are read by the Omega controller. The Omega controller delivers ON/OFF voltages to a processor (an Arduino UNO) after comparing the temperature detected inside the tank to a user input set point temperature $(77 \mathrm{~K})$. The Arduino utilizes these two level detecting sensor inputs and estimates the liquid filling valve state (open/close). The Arduino estimated valve state is fed to a solenoid valve with the aid of solid state relay (Crydom DC60S7). When the valve is open, it transfers the $\mathrm{LN}_{2}$ into the tank as shown in Figure 1.

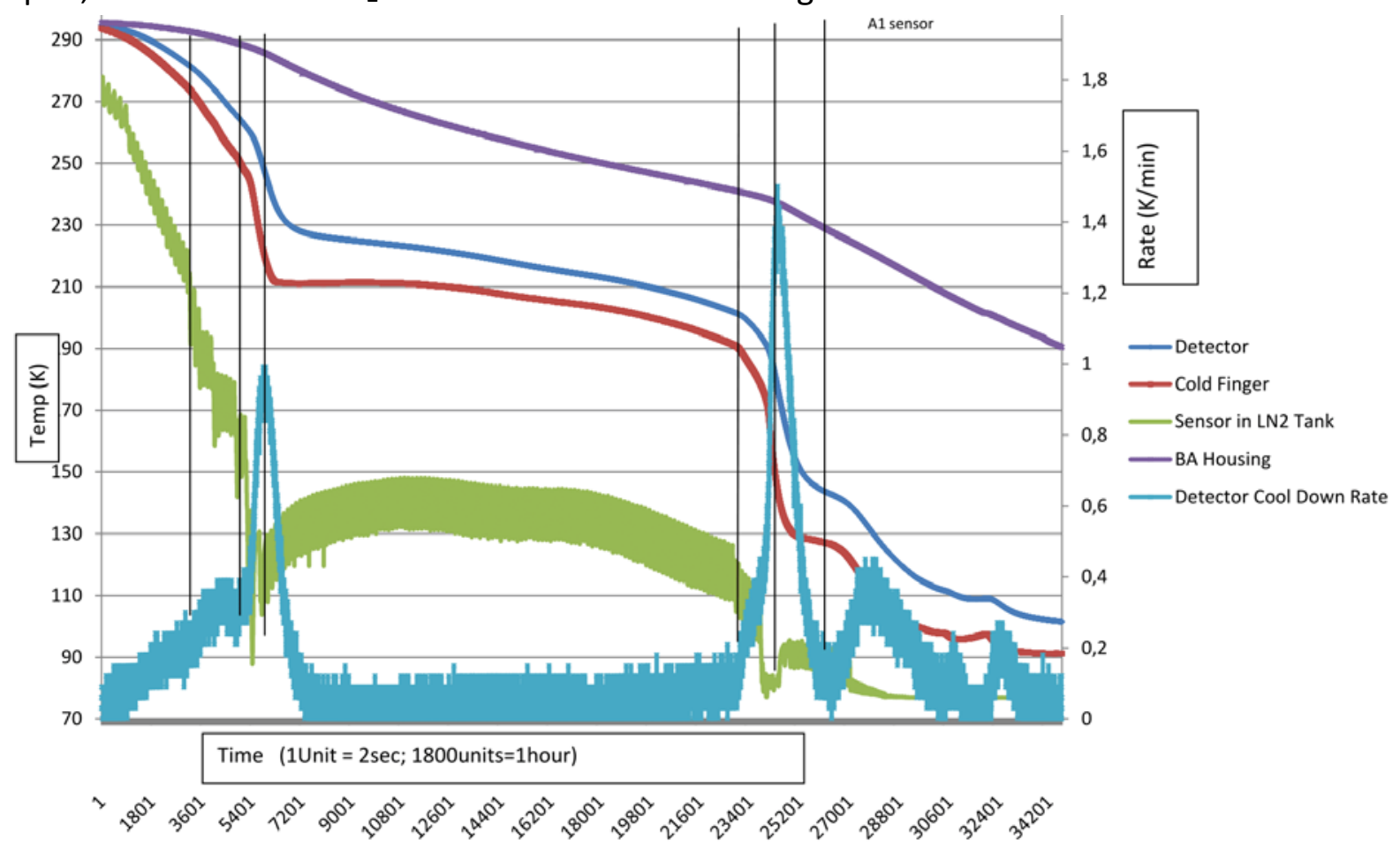

Figure 2: The temperatures of sensors attached at detector, cold finger, inside the $\mathrm{LN}_{2}$ tank and BA housing are presented. BA housing represents the core optics of acquisition camera. The detector cooled down/refrigeration rate is controlled to $2 \mathrm{~K} / \mathrm{min}$ limit

The detector temperatures are monitored using the ESO delivered Lakeshore cryogenic temperature monitor (model 224). This model only senses detector's temperature and it does not equip with heaters. Utilizing the Omega controller RS485 serial port communication, the liquid level detection sensors $\left(A_{0}\right.$ and $\left.A_{1}\right)$ and instrument temperatures are logged on a PC by using a FTDI device (USB-COM485-PLUS4).

\section{Control Software}

A Tcl-Tk and $\mathrm{C}$ combined software is written to read the temperatures of the level detection sensors $\left(A_{0}\right.$ and $\left.A_{1}\right)$ and detector's temperature for every $1 \mathrm{~s}$. The level detection sensors temperatures and detector temperature are acquired using Omega controller RS485 serial port communication and using a Lakeshore temperature monitor respectively. 
Using detector temperature for every $1 \mathrm{~s}$, the detector refrigeration rate $(\mathrm{K} / \mathrm{s})$ is computed and plugged into the Arduino software environment. Utilizing the both detector refrigeration rate and level detection sensors inputs, the Arduino software evaluates the final valve filling state.

In other approach, the valve is opened for $1 \mathrm{~min}$ and closed for 4 minutes to steadily refrigerate the detector by keeping refrigeration rate below $2 \mathrm{~K} / \mathrm{min}$. These two time periods are empirically calculated from a cooling down calibration as shown in Fig. 2. In this experiment different possibilities of time periods are kept under verification. From this, found out that 1 min opening and 4 min closing of valve are the best possibilities to auto fill the $\mathrm{LN}_{2}$ tank by maintaining the detector refrigeration rate below $2 \mathrm{~K} / \mathrm{min}$. While this fixed time period filling process is running, the tank is maintained without overflow. When the tank is full, the filling process is delayed to the next iteration of filling.

The software also handles the controller of failure cases. In case of the Dewar is run out of the $L N_{2}$, the software detects it by evaluating the inlet temperature sensor $\left(A_{1}\right)$ signals. Because the liquid level in the tank is being dropped down for every 1 second. In this case, the filling process is automatically terminated after checking 5 minutes of no liquid filling. The same case is repeated if any temperature sensors are broken for any reason.

In order to understand the system behavior in case of system malfunction and to quantify the instrument experimental performance at a desired temperature, the liquid level detection sensors delivered temperatures, the detector cooling rate, and liquid filling enabled instructions are logged and monitored for every $1 \mathrm{~s}$. These parameters are displayed in the lab using a graphical user interface as shown in Fig. 3. The instrument temperatures and detector cooling rate are plotted using Zabbix software and accessed remotely using a simple browser.

\section{Discussion}

The temperature controller developed in this work has successfully auto-filled and maintained the level of $\mathrm{LN}_{2}$ in between upper and lower limits as previously reported by Morrell and Manning 1996 and Murray and Atkinson 2004. Along with this, controller kept the detector refrigerate rate under control $(<2 \mathrm{~K} / \mathrm{min})$ during the cooling down process which is the critical requirement. It is the main and key outcome of the work and it is achieved with a low cost. So far, it is satisfactory with the performance in comparison to expensive commercially available controllers (example, Lakeshore model 350). This controller is successfully used for the acquisition camera optical performance validating experiments (Gordo et al. 2014) of GRAVITY project.

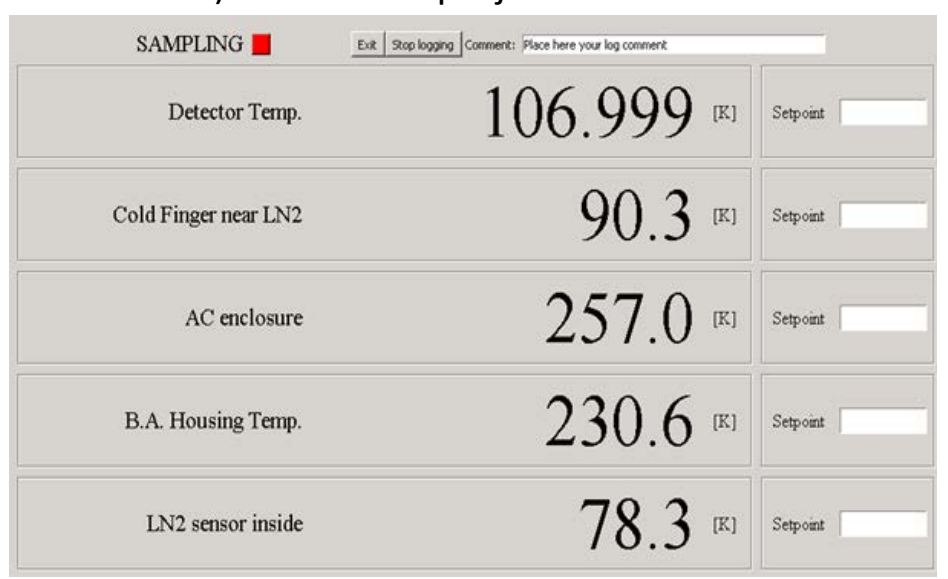

Figure 3: A graphical user interface (GUI) is developed to monitor the temperatures of different parts of the instrument during the cooling down process 
The fixed time and detector refrigeration rate limit are the critical parameters that need to be employed properly in order to refrigerate the system in a steady state and by keeping the detector refrigeration rate under control. The detector cooling rate feedback based controller is more efficient in performance in comparison to the fixed time period based controller. Reasons are the following: The rate of liquid transfer via valve is dependent on the pressure ( 0.5 bar) applied to the tank and the amount of liquid stored in it. When the fixed time period opening based controller is in operation, the liquid is pumped in large amounts in the beginning. But as the time goes on, the rate of liquid transferred by the valve is kept reduced and as a result the system gets warmer which is not desirable. Furthermore, the fixed time period controller requires a few calibration cool down experiments in the begging in order to choose the optimized fixed time value.

Some improvements can be developed to the current design. Currently the fixed time period is programmed in the beginning of control process. In order to address a uniform pumping rate during the cooling down process, it is envisaged to develop software which can accept flexible different fixed on-off timing lengths in the middle of the control process.

\section{Conclusions}

The developed controller is able to auto-fill the $\mathrm{LN}_{2}$ tank and also keeps the detector refrigeration rate under control during the cooling down process. This controller has withstood a large number of temperature cycles and it is reliable and efficient for our experiment. This controller is developed with a reasonable simple circuit and at low cost. It can be easily adaptable to research and student laboratory experiments.

Anugu acknowledges FCT for his PhD grant SFRH/BD/52066/2012. Thanks to J. Lima, J. Castanheira and A. Dias help in the laboratory. Special thanks to P. Carvas for helpful discussions. Authors would like to extend thanks to the anonymous reviewers for their valuable comments and suggestions to improve the manuscript.

\section{References}

Carvas, Pedro. 2012. "A Infra-Estrutura de Teste Da Câmara Multi-Telescópio Do GRAVITY." http://repositorio.ul.pt/handle/10451/9109.

Eisenhauer, F., G. Perrin, W. Brandner, C. Straubmeier, K. Perraut, A. Amorim, M. Schöller, et al. 2011. "GRAVITY: Observing the Universe in Motion." The Messenger 143: 16-24. http://adsabs.harvard.edu/abs/2011Msngr.143...16E.

Gamblin, Rodger L., Ernest Goldberg, and Dane T. Scag. 1959. "Liquid Nitrogen Liquid Level Controller." Review of Scientific Instruments 30 (5). AIP Publishing: 371. DOI: 10.1063/1.1716629.

Gordo, Paulo, Antonio Amorim, Jorge Abreu, Frank Eisenhauer, Narsireddy Anugu, Paulo Garcia, Oliver Pfuhl, et al. 2014. "Integration and Testing of the GRAVITY Infrared Camera for Multiple Telescope Optical Beam Analysis." In SPIE Astronomical Telescopes + Instrumentation, edited by Jayadev K. Rajagopal, Michelle J. Creech-Eakman, and Fabien Malbet, 91461V. International Society for Optics and Photonics. DOI: 10.1117/12.2056572.

Landis, D. A., N. W. Madden, and F. S. Goulding. 1986. "A Reliable Automatic Liquid Nitrogen Filling System." IEEE Transactions on Nuclear Science 33 (1). IEEE: 399-402. DOI: 10.1109/TNS.1986.4337129. 
Morrell, C., and P.P. Manning. 1996. "An Automatic Liquid-Nitrogen Filling System for Use with Solid-State X-Ray Detectors." Nuclear Instruments and Methods in Physics Research Section A: Accelerators, Spectrometers, Detectors and Associated Equipment 381 (2-3): 560-66. DOI: 10.1016/S0168-9002(96)00741-3.

Murray, Andrew J, and Steven Atkinson. 2004. "An Automatic Controller for Filling and Maintaining Liquid Nitrogen Levels in Dewars." Measurement Science and Technology 15 (5): N31-34. DOI: 10.1088/0957-0233/15/5/N01.

Shuhua Ma, Junjie Wang, Jinkuan Wang, Cheng Cui, and Yiding Zhao. 2010. "Research on the LN2 Auto-Filling System for Dewar Vessel Based on Bang-Bang + PID Control." In 2010 Chinese Control and Decision Conference, 2937-40. IEEE. DOI: 10.1109/CCDC.2010.5498670.

Zhao Yiding, and Song Zengfeng. 2010. "The Intelligent Controller of Filling Liquid Nitrogen Based on the Grey Prediction Theory." In 2010 International Conference On Computer Design and Applications, 3:V3-274 - V3-277. IEEE. DOI: 10.1109/ICCDA.2010.5540748. 